Petra Hauke, Madeleine Charney et Harri

Sahavirta : Going green: implementing sustainable

strategies in libraries around the world

\title{
Joachim Schöpfel
}

\section{(2) OpenEdition}

\section{Journals}

Édition électronique

URL : https://journals.openedition.org/edc/9601

DOI : 10.4000/edc. 9601

ISSN : 2101-0366

Éditeur

Université de Lille

Édition imprimée

Date de publication : 15 décembre 2019

Pagination : 213-216

ISBN : 978-2-917562-22-2

ISSN : 1270-6841

Référence électronique

Joachim Schöpfel, «Petra Hauke, Madeleine Charney et Harri Sahavirta : Going green: implementing sustainable strategies in libraries around the world », Études de communication [En ligne], 53 | 2019, mis en ligne le 15 mars 2020, consulté le 08 janvier 2022. URL : http://journals.openedition.org/edc/9601 ; DOl : https://doi.org/10.4000/edc.9601

Ce document a été généré automatiquement le 8 janvier 2022.

(c) Tous droits réservés 


\title{
Petra Hauke, Madeleine Charney et Harri Sahavirta : Going green: implementing sustainable strategies in libraries around the world
}

\author{
Joachim Schöpfel
}

\section{RÉFÉRENCE}

Hauke P., Charney M., Sahavirta H. (dir.) (2018). Going green: implementing sustainable strategies in libraries around the world. Berlin/Boston, De Gruyter Saur, IFLA Publication 177.

1 Le mouvement \#FridaysForFuture et les manifestations de la \#WeekForFuture fin septembre 2019 ont montré l'ampleur de la mobilisation globale pour le développement durable et contre le changement climatique. Comment contribuer à la protection de l'environnement? Comment participer au développement durable? Comment limiter les effets du réchauffement climatique? Dans le cadre de l'IFLA, les associations des bibliothécaires et documentalistes s'engagent depuis 2018 en faveur des objectifs de développement durable établis par les États membres des Nations Unies ${ }^{1}$. Il s'agit d'un engagement en lien avec le développement international de l'IFLA depuis 2016 (International Advocacy Program) $^{2}$ et de sa Déclaration de Lyon de 2014 sur l'accès à l'Information et au développement ${ }^{3}$.

2 À partir de 2009, un groupe de travail s'est formé au sein de l'IFLA et s'est fixé les objectifs suivants ${ }^{4}$ :

- analyser les effets du changement climatique sur les bibliothèques (modification des conditions de stockage et de conservation, isolation des bâtiments, impacts sur les finances et la gestion des bibliothèques...) ; 
- promouvoir l'application de pratiques respectueuses de l'environnement dans les bibliothèques (récupération des eaux de pluie, utilisation de sources d'énergie renouvelables, contrôle de l'impression, recyclage du papier, etc.) ;

- proposer des recommandations environnementales pour la profession (recyclage de documents périmés, utilisation de matériaux biodégradables, etc.) ;

- développer des ressources et des services de bibliothèque liés à la durabilité (développement de collections sur des thèmes environnementaux, expositions, sensibilisation, etc.), et sensibiliser des bibliothécaires aux préoccupations environnementales.

Ce groupe, du nom ENSULIB (Environment, Sustainability and Libraries Special Interest Group), organise des conférences et journées, maintient une liste de discussion, fait du community management et de la veille sur les réseaux sociaux, et décerne depuis 2016 un prix annuel pour des initiatives et réalisations modèles (IFLA Green Library Award ${ }^{5}$, sponsorisé par l'éditeur De Gruyter). Ont ainsi été récompensées des bibliothèques exemplaires dans plusieurs pays, dont la Stadtbibliothek Bad Oldesloe (Allemagne), la bibliothèque de Foshan (Chine) et, en 2019, la Biblioteca Pública Municipal Daniel Guillard à Cali (Colombie), pour ses projets innovateurs et son programme d'action sociale au sein de son quartier. La nouvelle médiathèque de Saint-Joseph sur l'île de la Réunion a fait partie du choix restreint, pour son bâtiment écologique extraordinaire.

ENSULIB diffuse également des recommandations, checklists et bilans d'actions, et a publié fin novembre un ouvrage collectif qui fait le point sur la préparation et la mise en œuvre de stratégies de développement durable dans les bibliothèques publiques et scientifiques. Coordonné par trois responsables du groupe ENSULIB, l'ouvrage positionne les bibliothèques comme acteurs-clés face à la crise écologique, avec une réponse "inclusive et globale ». L'objectif est de définir d'une manière exhaustive et précise ce que la prise de conscience environnementale signifie pour les bibliothèques.

L'ouvrage contient trois sections. Les cinq chapitres de la première partie proposent un cadre général autour d'une synthèse d'initiatives modèles, y compris pour évaluer les projets et pour développer une stratégie de management durable. La conviction partagée est qu'il ne suffit pas d'un toit végétalisé pour transformer une bibliothèque en "bibliothèque verte ", respectant l'environnement et le développement durable ; qu'un projet durable ne coûte pas nécessairement cher; qu'il s'agit d'une approche globale $\mathrm{du}$ fonctionnement d'une bibliothèque; que toutes les bibliothèques sont concernées ; et qu'il s'agit d'enjeux et d'un engagement bien au-delà du périmètre de l'institution.

6 La deuxième partie contient huit études de cas d'une stratégie de développement durable mise en œuvre dans des bibliothèques publiques, avec des exemples de la Suède, du Kenya, de l'Ukraine, de la Chine, de la Serbie ou de l'Allemagne. La troisième partie, consacrée aux bibliothèques scientifiques, rassemble cinq études de cas de réalisations dans des bibliothèques universitaires de Hong Kong, du Cameroun, d'Allemagne, d'Ouganda et du Kenya. Pourquoi ce choix ? Il s'agit en fait des meilleurs articles de la réunion satellite ENSULIB 2017 à Berlin, de la session de la section ENSULIB/bibliothèques publiques à la conférence IFLA 2017 en Pologne et du prix IFLA Green Library Award 2017.

7 On peut regretter l'absence d'une étude de cas sur l'une des bibliothèques vertes en France. Pourtant, le mémoire d'étude de Manon Le Guennec $(2018)^{6}$, primé par les palmes de l'Enssib, avait fait l'inventaire d'actions emblématiques dans plusieurs bibliothèques et médiatiques. Mais elle avait aussi posé la question du positionnement 
des bibliothèques et de l'écologie comme levier de communication et de promotion. Le mémoire mentionnait notamment la mobilisation de l'Association des Bibliothécaires de France $(\mathrm{ABF})$, de la $\mathrm{Bpi}$, du Comité français international bibliothèques et documentation (Cfibd) et de l'Enssib pour recenser et valoriser l'action des bibliothèques et CDI en faveur de l'environnement et de l'Agenda 2030, le programme de l'ONU pour le développement durable. Le site de cette initiative est désormais en ligne ${ }^{7}$, et le 25 septembre dernier, date anniversaire de l'Agenda 2030, a vu la mobilisation de plus de 1600 bibliothèques municipales, départementales, universitaires et scolaires (CDI) à travers toute la France, en faveur du développement durable.

8 Revenons à l'ouvrage collectif du groupe ENSULIB. Tous les articles sont écrits en anglais. Rédigés d'une manière claire, et pour certains avec des illustrations, ils montrent d'une manière exemplaire comment une bibliothèque peut devenir acteur du développement durable, avec les facteurs-clés pour réussir, mais aussi les obstacles et impasses. Il s'agit d'un ouvrage écrit par des bibliothécaires à destination de leurs collègues, un retour d'expérience collectif, discuté, évalué et sélectionné, destiné à enrichir les débats au sein des associations et institutions.

La suite est déjà en chantier. Le groupe ENSULIB prépare, avec la section "Library buildings and equipment » de l'IFLA, un nouveau livre sur les enjeux de la transformation écologique de bâtiments anciens (historiques) pour héberger des bibliothèques, qui paraîtra en 2020 chez De Gruyter Saur dans la collection IFLA. Peut-être y aura-t-il un jour un ouvrage un peu plus conceptuel, avec une analyse de l'impact de cette mobilisation sur les missions traditionnelles, sur les dispositifs et les formes de médiatisation des bibliothèques.

Pour finir, depuis l'été 2019 il existe, en réaction au mouvement \#FridaysForFuture, une initiative \#Libraries4Future ${ }^{8}$ pour lancer et coordonner une mobilisation internationale des bibliothécaires, partant du principe que les bibliothèques sont des acteurs de la protection du climat, que tout le monde peut et doit agir, que les bibliothèques sont des multiplicateurs, des espaces d'éducation et d'information, et qu'elles renforcent la cohésion sociale. L'ouvrage collective dirigé par Petra Hauke, Madeleine Charney et Harri Sahavirta donne de précieux exemples et conseils pour concrétiser cet engagement sur le terrain.

\section{NOTES}

1. https://www.ifla.org/libraries-development.

2. https://www.ifla.org/ldp/iap.

3. https://www.lyondeclaration.org/.

4. https://www.ifla.org/about-environmental-sustainability-and-libraries.

5. https://www.ifla.org/node/10159.

6. Le Guennec M. (2018). Bibliothèques et écologie : Les bibliothèques de lecture publique françaises et les enjeux environnementaux. Mémoire d'étude de Conservateur des bibliothèques, Enssib. Disponible sur https://www.enssib.fr/bibliotheque-numerique/notices/68275-bibliotheques-et-ecologie-les- 
bibliotheques-de-lecture-publique-francaises-et-les-enjeux-environnementaux (page consultée le 29 octobre 2019).

7. https://agenda2030bibfr.wixsite.com/agenda2030bib.

8. https://libraries4future.org/petition/.

\section{AUTEURS}

JOACHIM SCHÖPFEL

Univ. Lille, EA 4073 - GERiiCO, F-59000 Lille, France

joachim.schopfel@univ-lille.fr 